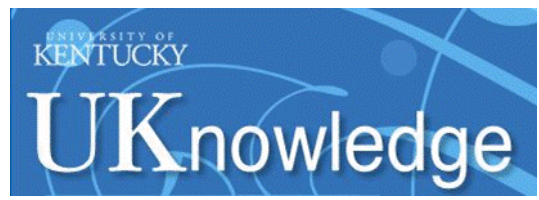

University of Kentucky

UKnowledge

\title{
SGLT2 Inhibitor Therapy Improves Blood Glucose but Does Not Prevent Diabetic Bone Disease in Diabetic DBA/2J Male Mice
}

\author{
Kathryn M. Thrailkill \\ University of Kentucky, kathryn.thrailkill@uky.edu \\ R. Clay Bunn \\ University of Kentucky, clay.bunn@uky.edu \\ Jeffry S. Nyman \\ Vanderbilt University \\ Mallikarjuna R. Rettiganti \\ University of Arkansas for Medical Sciences \\ Gael E. Cockrell \\ University of Arkansas for Medical Sciences
}

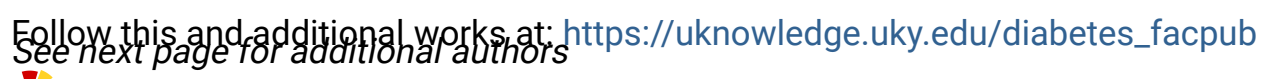

Part of the Chemicals and Drugs Commons, Diseases Commons, and the Endocrinology, Diabetes, and Metabolism Commons

Right click to open a feedback form in a new tab to let us know how this document benefits you.

\section{Repository Citation}

Thrailkill, Kathryn M.; Bunn, R. Clay; Nyman, Jeffry S.; Rettiganti, Mallikarjuna R.; Cockrell, Gael E.; Wahl, Elizabeth C.; Uppuganti, Sasidhar; Lumpkin, Charles K.; and Fowlkes, John L., "SGLT2 Inhibitor Therapy Improves Blood Glucose but Does Not Prevent Diabetic Bone Disease in Diabetic DBA/2J Male Mice" (2016). Barnstable Brown Diabetes Center Faculty Publications. 5.

https://uknowledge.uky.edu/diabetes_facpub/5

This Article is brought to you for free and open access by the Diabetes at UKnowledge. It has been accepted for inclusion in Barnstable Brown Diabetes Center Faculty Publications by an authorized administrator of UKnowledge. For more information, please contact UKnowledge@lsv.uky.edu. 
SGLT2 Inhibitor Therapy Improves Blood Glucose but Does Not Prevent Diabetic Bone Disease in Diabetic DBA/2J Male Mice

Digital Object Identifier (DOI)

https://doi.org/10.1016/j.bone.2015.07.025

Notes/Citation Information

Published in Bone, v. 82, p. 101-107.

Copyright $@ 2015$ Elsevier Inc. All rights reserved.

This manuscript version is made available under the CC-BY-NC-ND 4.0 license

https://creativecommons.org/licenses/by-nc-nd/4.0/.

The document available for download is the author's post-peer-review final draft of the article.

A corrigendum to this article can be found at: https://doi.org/10.1016/j.bone.2016.11.021

\section{Authors}

Kathryn M. Thrailkill, R. Clay Bunn, Jeffry S. Nyman, Mallikarjuna R. Rettiganti, Gael E. Cockrell, Elizabeth C. Wahl, Sasidhar Uppuganti, Charles K. Lumpkin, and John L. Fowlkes 
Published in final edited form as:

Bone. 2016 January ; 82: 101-107. doi:10.1016/j.bone.2015.07.025.

\title{
SGLT2 inhibitor therapy improves blood glucose but does not prevent diabetic bone disease in diabetic DBA/2J male mice
}

\author{
Kathryn M. Thrailkill, MD ${ }^{\mathrm{a}}$, R. Clay Bunn, $\mathrm{PhD}^{\mathrm{a}}$, Jeffry S. Nyman, $\mathrm{PhD}^{\mathrm{b}}$, Mallikarjuna R. \\ Rettiganti, PhD ${ }^{c}$, Gael E. Cockrell ${ }^{\mathrm{C}}$, Elizabeth C. Wahl' ${ }^{\mathrm{C}}$, Sasidhar Uppuganti ${ }^{\mathrm{b}}$, Charles K. \\ Lumpkin Jr., PhD' , and John L. Fowlkes, MD ${ }^{a}$ \\ aUniversity of Kentucky Barnstable Brown Diabetes and Obesity Center and the Department of \\ Pediatrics, University of Kentucky College of Medicine, Lexington, KY 40536 \\ bVA Tennessee Valley Health Care System, Department of Orthopaedic Surgery \& Rehabilitation \\ and Center for Bone Biology, Vanderbilt University Medical Center, Nashville, TN 37232 \\ 'Department of Pediatrics, University of Arkansas for Medical Sciences and Arkansas Children's \\ Hospital Research Institute, Little Rock, AR, 72202, USA
}

\section{Abstract}

Persons with type 1 and type 2 diabetes have increased fracture risk, attributed to deficits in the microarchitecture and strength of diabetic bone, thought to be mediated, in part, by the consequences of chronic hyperglycemia. Therefore, to examine the effects of a glucose-lowering SGLT2 inhibitor on blood glucose (BG) and bone homeostasis in a model of diabetic bone disease, male DBA/2J mice with or without streptozotocin (STZ)-induced hyperglycemia were fed chow containing the SGLT2 inhibitor, canagliflozin (CANA), or chow without drug, for 10 weeks of therapy. Thereafter, serum bone biomarkers were measured, fracture resistance of cortical bone was assessed by $\mu \mathrm{CT}$ analysis and a three-point bending test of the femur, and vertebral bone strength was determined by compression testing. In the femur metaphysis and L6 vertebra, longterm diabetes (DM) induced deficits in trabecular bone microarchitecture. In the femur diaphysis, a decrease in cortical bone area, cortical thickness and minimal moment of inertia occurred in DM $(\mathrm{p}<0.0001$, for all) while cortical porosity was increased $(\mathrm{p}<0.0001)$. These DM changes were associated with reduced fracture resistance (decreased material strength and toughness; decreased structural strength and rigidity; $\mathrm{p}<0.001$ for all). Significant increases in PTH $(\mathrm{p}<0.0001)$,

Corresponding Author: Kathryn M. Thrailkill, MD, University of Kentucky, Department of Pediatrics, Kentucky Clinic, 740 S. Limestone, Lexington, KY 40536, kathryn.thrailkill@uky.edu.

Present Address: Barnstable Brown Diabetes and Obesity Center and Department of Pediatrics, University of Kentucky College of Medicine, 740 S. Limestone St., Lexington, KY, USA 40536.

Former Address: Department of Pediatrics, University of Arkansas for Medical Sciences, \#1 Children's Way, Little Rock, AR, USA, 72202.

Publisher's Disclaimer: This is a PDF file of an unedited manuscript that has been accepted for publication. As a service to our customers we are providing this early version of the manuscript. The manuscript will undergo copyediting, typesetting, and review of the resulting proof before it is published in its final citable form. Please note that during the production process errors may be discovered which could affect the content, and all legal disclaimers that apply to the journal pertain.

Author Contributions: K.M.T., R.C.B., C.K.L and J.L.F. contributed to the design of the experiment. K.M.T., R.C.B., J.S.N. and J.L.F. researched data, contributed to discussion, wrote manuscript and reviewed/edited manuscript. G.E.C, E.C.W and S.U. researched data and/or reviewed/edited manuscript. M.R.R provided statistical analyses and reviewed/edited manuscript. C.K.L. also reviewed/edited the manuscript.

Conflict of Interest: The authors have no financial or personal conflicts of interest to disclose. 
RatLAPs ( $\mathrm{p}=0.0002)$, and urine calcium concentration $(\mathrm{p}<0.0001)$ were also seen in DM. Canagliflozin treatment improved BG in DM mice by $\sim 35 \%$, but did not improve microarchitectural parameters. Instead, in canagliflozin-treated diabetic mice, a further increase in RatLAPs was evident, possibly suggesting a drug-related intensification of bone resorption. Additionally, detrimental metaphyseal changes were noted in canagliflozin-treated control mice. Hence, diabetic bone disease was not favorably affected by canagliflozin treatment, perhaps due to insufficient glycemic improvement. Instead, in control mice, long-term exposure to SGLT2 inhibition was associated with adverse effects on the trabecular compartment of bone.

\section{Keywords}

Diabetic bone disease; type 1 diabetes; trabecular bone; cortical bone; microarchitecture; canagliflozin

\subsection{Introduction}

Osteopenia, osteoporosis and risk for fracture are all increased in persons with type 1 diabetes (T1D), with a propensity for fracture that is greater than would be predicted by bone mineral density (BMD) alone $(1,2)$. Hence, the impact of chronic hyperglycemia on bone quality and bone microarchitecture is relevant to diabetic bone disease. Renal glucose resorption is regulated by the sodium glucose co-transporter 2 (SGLT2), a high affinity transporter expressed in the proximal tubule. SGLT2 inhibitors, by inhibiting reabsorption of filtered glucose in the renal proximal tubule, lower blood glucose levels in persons with diabetes, independent of insulin action or secretion and without inducing hypoglycemia (3). Consequently, they have been shown to improve HbAlc in persons with type 2 diabetes (T2D) (3). Currently, T1D is not a Food and Drug Administration (FDA)-approved indication for SGLT2 inhibitor therapy, although registered clinical trials in T1D are underway (ClinicalTrials.gov Identifier:NCT02139943), and are supported by proof-ofconcept trials which demonstrate glycemic improvement from SGLT2 adjunctive-to-insulin treatment in T1D (4), as well as from dual SGLT1/SGLT2 inhibitor adjunctive-to-insulin treatment in T1D (5). Early clinical investigation in T2D, however, has demonstrated a possible $\sim 30 \%$ increase in bone fractures in patients receiving the SGLT2 inhibitor, canagliflozin (6). Therefore, using a mouse model of diabetic bone disease which is characterized by insulin deficiency (i.e., streptozotocin-induced diabetes), we examined the effects of 10 weeks of treatment with the SGLT2 inhibitor, canagliflozin (CANA) on bone microarchitecture and material strength of the appendicular and axial skeleton, as a preclinical investigation of the skeletal impact of SGLT2 inhibitor therapy in T1D.

\subsection{Materials and Methods}

\subsubsection{Animal model}

The DBA/2J strain of mice (The Jackson Laboratories, Bar Harbor, Maine) exhibits a propensity for the development of diabetic complications (7), and as we have previously shown, develops a diabetic bone phenotype within 10 weeks of hyperglycemia (8). Male DBA/2J mice, 10-11 weeks of age, were treated either with streptozotocin (STZ) to induce 
diabetes $(40 \mathrm{mg} / \mathrm{kg} / \mathrm{day} \times 5$ days) or with vehicle (100 mM citrate, $\mathrm{pH} 4.5)$. At $\sim 12-13$ weeks of age, diabetic mice, as confirmed by glucosuria, were then randomly assigned to treatment groups as follows; DBA/2J mice with streptozotocin (STZ)-induced diabetes were either fed Teklad 8640 chow containing the SGLT2 inhibitor, canagliflozin (STZ+CANA, $50 \mathrm{ppm}, \mathrm{n}=19$ ) or chow without canagliflozin (STZ+VEHICLE, $\mathrm{n}=18$ ). Non-diabetic (control) mice were fed chow containing a slightly higher canagliflozin concentration (CONT+CANA, $62.5 \mathrm{ppm}$; to approximately offset the polyphagia in diabetic mice; $\mathrm{n}=20$ ), or chow without canagliflozin (CONT+VEHICLE, $n=20$ ). Mice were provided ad libitum access to water and to their assigned food for the next 10 weeks. Based on differing rates of food consumption between diabetic and non-diabetic animals, canagliflozin intake during the 10 week treatment period was $17.7-20.6 \mathrm{mg} / \mathrm{kg} /$ day (mean $\pm \mathrm{SD} ; 18.7 \pm 1.0$ ) in the STZ (diabetes) group and $14.2-19.6 \mathrm{mg} / \mathrm{kg} /$ day $(16.6 \pm 2.0)$ in the non-diabetes group. Blood glucose was measured at 3 and 10 weeks of treatment, via glucometer (OneTouch ${ }^{\circledR}$ Ultra ${ }^{\circledR 2}$ Blood Glucose Monitoring System, Lifescan, Inc., Milpitas, CA; average intra-assay coefficient of variation, $1.7 \%$ across a range of $40-300 \mathrm{mg} / \mathrm{dL}$ target glucose concentrations). All procedures were approved by the Institutional Animal Care and Use Committee at the University of Arkansas for Medical Sciences.

\subsubsection{Skeletal Assessment}

After euthanasia, the left femur was harvested and femur length was measured using calipers. Bones [femurs (all mice) and L6 vertebra ( $\mathrm{n}=10$ mice per group)] were frozen in phosphate buffered saline (PBS) and stored at $-20^{\circ} \mathrm{C}$ until analysis. With each femur immersed in PBS at room temperature, the long axis of the bone was scanned with a microcomputed tomography $(\mu \mathrm{CT})$ scanner (Scanco Medical $\mu \mathrm{CT} 40$, Bröttisellen, Switzerland): voxel size of $12 \mu \mathrm{m}$, energy settings of $70 \mathrm{kVp} / 0.114 \mathrm{~mA}$, integration time of $300 \mathrm{~ms}$ with 1000 projections per full rotation, and calibrated to a hydroxyapatite (HA) phantom, as we have previously described (7). Likewise, the cranial-caudal axis of each L6 vertebral body (VB) was aligned with the long axis of the specimen tube and scanned while immersed in PBS using another $\mu C T$ scanner (Scanco Medical $\mu$ CT50, Bröttisellen, Switzerland): voxel size of $10 \mu \mathrm{m}$, energy settings of $55 \mathrm{kVp} / 0.20 \mathrm{~mA}$, integration time of $1200 \mathrm{~ms}$ with 1000 projections per full rotation, and calibration to a HA phantom. For the femur, the regions of interest (ROI) included the trabecular bone of the metaphysis and the cortical bone of the diaphysis (1.2 $\mathrm{mm}$ in length for both compartments). For the VB, the ROI included the trabecular bone between the end-plates and within the cortical shell. Each ROI had a unique threshold $\left(465.7 \mathrm{mgHA} / \mathrm{cm}^{3}\right.$ and $912.6 \mathrm{mgHA} / \mathrm{cm}^{3}$ for trabecular and cortical bone of the femur, respectively and $304.3 \mathrm{mgHA} / \mathrm{cm}^{3}$ for trabecular bone of the VB) and Gaussian noise filter (sigma of 0.2 with support of 1 for both trabecular and cortical bone) used for all scans. Standard evaluation scripts from the manufacturer were used to determine the architectural and structural properties of trabecular and cortical bone, respectively.

\subsubsection{Biomechanical Testing}

To determine mechanical properties of cortical bone, each hydrated femur or each hydrated VB was loaded to failure at $3 \mathrm{~mm} / \mathrm{min}$ using a three-point bending fixture (bending about the medial-lateral plane) or using compression platens with a moment relief, respectively. The span between the lower supports was $8 \mathrm{~mm}$. From the resulting forces recorded by a 
$100 \mathrm{~N}$ load cell (Honeywell; Morristown, NJ) and displacements recorded by the LVDT (Dynamight 8841, Instron; Canton, $\mathrm{OH}$ ) structural properties included the initial stiffness and the peak force endured by the femur. Material properties of modulus and strength of the cortex were also estimated using standard beam theory. The $\mu \mathrm{CT}$ scans provided the moment of inertia $\left(\mathrm{I}_{\mathrm{min}}\right)$ and the distance between the neutral axis of bending and the outermost point in the anterior-posterior direction $\left(\mathrm{C}_{\mathrm{min}}\right)$. For the compression tests, we recorded the peak force as the measurement of whole-bone strength of the VB (Force) and divided the peak force by the mean estimated cross-sectional area as the measurement of the apparent strength of the VB (Stress).

\subsubsection{Bone Biomarker Analyses}

For all mice in all groups, procollagen type $1 \mathrm{~N}$-terminal propeptide (P1NP), a marker of bone formation, was measured in serum at sacrifice, using the Rat/Mouse P1NP Enzyme immunoassay (Immunodiagnostics Systems, Inc., Fountain Hills, AZ; \#AC-33F1); Cterminal telopeptides of type I collagen (RatLAPs), a marker of bone resorption, was measured in serum using the RatLAPs ELISA (Immunodiagnostics Systems, Inc., Fountain Hills, AZ; \#AC-06F1). Due to limitations in serum availability, for a smaller subset of mice (STZ+CANA, n=10; STZ+VEH, n=13; CONT+VEH, n=17), osteoclast-derived, tartrateresistant acid phosphatase (TRACP 5b) activity, a marker of osteoclast number, was measured. TRACP $5 \mathrm{~b}$ activity was measured using the MouseTRAP ${ }^{\mathrm{TM}}$ ELISA (Immunodiagnostics Systems, Inc., Fountain Hills, AZ; \#SB-TR103).

To assess the consequences of SGLT2-induced osmotic diuresis on urinary mineral loss, we also examined select components of calcium and phosphate homeostasis. Using urine collected from each animal within one week of sacrifice, urine calcium was measured using a calcium colorimetric assay (Sigma-Aldrich Corporation, St. Louis, MO; \#MAK022) and urine creatinine was measured using an alkaline picrate chemical assay (Exocell, Inc., Philadelphia, PA; The Creatinine Companion, \#1012). Calcium concentration was then normalized to urine creatinine concentration, and reported as a calcium/creatinine ratio (CCR). Mouse intact parathyroid hormone (PTH 1-84), indicative of serum calcium and phosphate regulation, was measured in plasma at sacrifice using the mouse two-site ELISA (Alpco Diagnostics, Salem, NH; \#31-PTHMS-E01). FGF23, a bone-derived marker of chronic kidney disease that functions to inhibit renal reabsorption of phosphate, independent of PTH (8), was measured using the MILLIPLEX MAP Mouse Bone Magnetic Bead kit (\# MBNMAG-41K) for FGF23.

\subsubsection{Quantitative RT-PCR}

To quantify disease or drug-induced changes in renal expression of genes involved in calcium and phosphate regulation, quantitative RT-PCR was performed essentially as described by Fowlkes, et al, (9) using the following primers: 25-hydroxyvitamin D-1 alpha hydroxylase (CYP27B1), forward: 5' acggcggatggtgaagaatg 3', reverse: 5' ttgtccagagttccagcatagc $3^{\prime}$; and the solute carrier family 34 (type II sodium/phosphate cotransporter, member 1, SLC34A1), forward: 5' caacatctcgggcatcctactg 3', reverse: 5' ggcaagagcaggaagcacac $3^{\prime}$. Target mRNA expression was normalized to actin expression to 
control for cDNA loading variation (actin primers: forward: 5' tctggcaccacaccttctaca 3', reverse: 5' cagccaggtccagacgcagga 3').

\subsubsection{Statistical Analyses}

All data were checked for inconsistencies and outliers. Highly influential observations were removed prior to analysis. A one-way analysis of variance (ANOVA) was performed to test whether the means of several bone parameters were equal between the four groups: STZ +CANA, STZ+VEH, CONT+CANA, CONT+VEH. Linear contrasts for the group variable were used to test the following four pairwise comparisons: STZ+CANA vs. STZ+VEH, CONT+CANA vs. CONT+VEH, STZ+CANA vs. CONT+CANA, and STZ+VEH vs. CONT+VEH. For each parameter, a step-down Bonferroni method was used to keep the overall family-wise error rate under 0.05 to adjust for multiple comparisons.

Pearson's correlation was used to test whether there was any association between PTH, P1NP, RatLAPs, FGF23, and CCR with other skeletal (diaphysis and metaphysis) parameters. Variables violating the normality assumption were log-transformed prior to analysis. All tests were two-sided assuming a significance level of 5\%. All statistical analyses were generated using SAS/STAT software, Version 9.4 of the SAS System for Windows 7, Copyright (C) 2002-2012 SAS Institute Inc.

\subsection{Results}

Changes in body weight and blood glucose across the ten-week treatment period are shown in Table 1. Selected bone parameters, as determined by $\mu \mathrm{CT}$ and biomechanical testing, are also shown in Table 1. Representative $\mu \mathrm{CT}$ images are shown in Figure 1. Serum and urine bone biomarker concentrations are demonstrated in Figure 2. Relevant gene expression is compared in Figure 3.

\subsubsection{Metabolic effects}

Throughout 10 weeks of canagliflozin treatment, body weights and blood glucose levels were not significantly different between the two control groups (Table 1: CONT+CANA vs. $\mathrm{CONT}+\mathrm{VEH})$. In contrast, both groups of diabetic mice (STZ+CANA, STZ+VEH) exhibited hyperglycemia and weight loss, relative to their non-diabetic counterparts.

\subsubsection{Effect of diabetes on bone microarchitecture, material and structural qualities of the appendicular skeleton}

When examining bone quality, consistent with our previous findings (10), long-term diabetes was associated with deficits in trabecular bone microarchitecture in the femur (Table 1: Diaphysis, Metaphysis; P4), including decreased trabecular bone volume fraction (BV/TV), trabecular number (Tb.N), trabecular thickness (Tb.Th) and tissue mineral density (Tb.TMD), along with increased trabecular spacing (Tb.Sp; $\mathrm{p}<0.0001$ for all parameters) and changes in trabecular shape (SMI; $\mathrm{p}=0.002$ ). Deficits in cortical bone content were also evident, characterized by decreased cortical area (Ct.AR, $\mathrm{p}<0.0001$; Tt.AR, $\mathrm{p}=0.004$ ) and cortical thickness (Ct.Th; $\mathrm{p}<0.0001$ ), coupled with an increase in cortical porosity $(\mathrm{p}<0.0001)$. These DM changes were associated with a reduced resistance to fracture. 
Specifically, with respect to material properties, bending strength and toughness were decreased in diabetic mice. As for whole-bone, structural properties, specifically structural strength (peak moment) and stiffness (rigidity) were lower for diabetic than for non-diabetic mice ( $\mathrm{p}<0.0001$ for all). These deficits in bone structure are depicted in representative $\mu \mathrm{CT}$ images (Figure 1B, compared with 1D).

\subsubsection{Effects of diabetes on bone microarchitecture and strength of the axial skeleton}

We have not previously reported on vertebral bone strength and quality in this model. Analysis of the L6 vertebra, as shown in Table 1, demonstrated that long-term diabetes was also associated with deficits in trabecular microarchitecture (significant decreases in Tb.BV/TV and Tb.TMD). Moreover, diabetic vertebrae were significantly less resistant to fracture by compression testing (Force).

\subsubsection{Effects of diabetes on bone biomarkers}

Measurements of P1NP, a marker of bone formation, were no different between diabetic and control groups (Figure 2B). However, secondary hyperparathyroidism (Figure 2A; $\mathrm{p}<0.0001$ ), along with significant increases in urine calcium concentration (Table 1; CCR; $\mathrm{p}<0.0001$ ), and serum RatLAPs (a marker of bone resorption) (Figure 2C; $\mathrm{p}=0.0002$ ) were all evident in DM.

TRACP 5b (a marker of osteoclast number) was also increased in DM (STZ+VEH, $12.0 \pm$ 3.0 U/L vs. CONT+VEH, $9.8 \pm 1.6 \mathrm{U} / \mathrm{L}, \mathrm{p}=0.06$; $\mathrm{STZ}+\mathrm{CANA}, 13.5 \pm 4.3 \mathrm{U} / \mathrm{L}$ vs. CONT +CANA, $9.8 \pm 1.4 \mathrm{U} / \mathrm{L}, \mathrm{p}=0.002$ ) and the overall effect of disease on TRACP $5 \mathrm{~b}$ was significant $(\mathrm{p}<0.0001)$; however, perhaps due to the reduced sample size for this particular assay, the increase was not significant when comparing STZ+VEH with CONT+VEH $(\mathrm{p}=0.06)$.

Regarding biomarkers that were significantly increased in DM, RatLAPs was negatively associated with Ct.Th ( $\mathrm{r}=-0.56 ; \mathrm{p}=0.02)$, Ct.TMD ( $\mathrm{r}=-0.59 ; \mathrm{p}=0.01)$, Tb.Th $(\mathrm{r}=-0.49$; $\mathrm{p}=0.04)$, Tb.TMD $(r=-0.49 ; \mathrm{p}=0.05)$ and peak moment $(\mathrm{r}=-0.53 ; \mathrm{p}=0.03)$ in untreated diabetic mice (STZ+VEH). CCR was negatively associated with Ct.TMD $(r=-0.52 ; \mathrm{p}=0.03)$ and Tb.N ( $r=-0.56 ; p=0.02)$, while being positively associated with Tb.Sp $(r=0.58 ; p=0.01)$. Serum PTH, however, was not specifically related to cortical or trabecular bone parameters, either in untreated diabetic mice (STZ+VEH), or in both diabetic groups when combined (STZ+VEH, STZ+CANA), suggesting that differences in PTH did not explain the differences in bone phenotype.

\subsubsection{Effects of Canagliflozin treatment in diabetic and non-diabetic mice}

Consistent with treatment expectations, canagliflozin intake by diabetic mice decreased mean blood glucose levels from $520.94 \pm 84.69 \mathrm{mg} / \mathrm{dL}$ to $358.84 \pm 72.3 \mathrm{mg} / \mathrm{dL}$ after 3 weeks and from $525.18 \pm 53.64 \mathrm{mg} / \mathrm{dL}$ to $338.89 \pm 131.45 \mathrm{mg} / \mathrm{dL}$ after 10 weeks of treatment (mean $\pm \mathrm{SD} ; \mathrm{p}<0.0001$ for both comparisons). This glucose improvement was not, however, associated with recovery of any of the trabecular or cortical microarchitecture parameters [Table 1 (P1); Figure 1A vs. 1B]. Moreover, canagliflozin treatment did not prevent the diabetes-related decrease in the fracture resistance of either the femur or L6 
vertebra [Table 1 (P1, P3)]. Specifically, bending strength, toughness, peak moment, rigidity, and force were all lower for canagliflozin-treated diabetic mice, compared with canagliflozin-treated non-diabetic mice. However, when comparing the STZ+CANA and STZ+VEH groups, a further increase in serum RatLAPs was evident in canagliflozin-treated diabetic mice (Figure 2C).

Canagliflozin treatment of control mice did not significantly alter blood glucose levels or body weight (Table 1). However, canagliflozin treatment of control mice did reduce femoral trabecular BV/TV, Tb.N and Tb.TMD, while increasing Tb.SP (Table 1, P2), suggesting detrimental drug-induced metaphyseal changes in control animals. Urine calcium excretion $(\mathrm{CCR} ; \mathrm{p}=0.003)$ in canagliflozin-treated control mice was also significantly increased, as were levels of serum FGF23 (a regulator of urine phosphate excretion; Figure 2D, p=0.046), possibly suggesting a subclinical but compensatory hyperparathyroidism in this group, in response to increased urine calcium losses.

\subsubsection{CYP27B1 and SLC34A1 gene expression}

Differences in renal gene expression of CYP27B1 and SLC34A1 were evident between diabetic and non-diabetic mice. Specifically, expression of CYP27B1 mRNA was increased by $\sim 11$-fold $(\mathrm{p}<0.0001)$ and expression of SLC34A1 was decreased by $\sim 30 \%(\mathrm{p}=0.046)$ in diabetic mice (STZ+VEH) versus non-diabetic mice (CON+VEH) (Figure 3). Canagliflozin treatment reduced by $50 \%$ the expression of CYP27B1 mRNA in diabetic mice (STZ +CANA vs. STZ+VEH, p=0.012), but did not change SLC34A1 expression. Canagliflozin treatment of control mice did not significantly impact the expression of either gene.

\subsection{Discussion}

Increased bone fragility, contributing to increased fracture risk, is a significant comorbidity in persons with either type 1 diabetes (T1D) or type 2 diabetes (T2D); and is likely the consequence of many common variables, including chronic hyperglycemia, tissue-specific accumulation of advanced glycation end-products (AGEs) and secondary hyperparathyroidism. The SGLT2 inhibitor class of drugs enhances urinary glucose excretion by inhibiting the function of the renal sodium-glucose cotransporter- 2 in the early proximal convoluted tubule. While beneficial for the treatment of hyperglycemia, the excess $\mathrm{Na}^{+}$in the tubular lumen can alternatively be reabsorbed via the $\mathrm{Na}^{+}$-Phosphate transporter, thereby increasing serum phosphate concentration. The expected compensatory physiological response would include an increase in both parathyroid hormone (PTH) and fibroblast growth factor 23 (FGF23), leading to phosphaturia and a return to normophosphatemia. Additionally, chronic glucosuria, both as a consequence of uncontrolled diabetes mellitus, or as augmented by SGLT2 inhibitor therapy, contributes to diabetic hypercalciuria via osmotic diuresis (11-13). Analogously, hypercalciuria is a reported comorbidity in some children with severe Familial Renal Glucosuria, a disorder attributed to mutations in the gene for SGLT2 (slc5a2) (14), suggesting that SGLT2 drug exposure might augment urinary calcium loss in diabetes, despite improving glycemic control. Taken together, a "perfect storm" of factors detrimental to bone mineral homeostasis could exist, particularly in patients receiving long-term treatment with SGLT2 inhibitors. 
The present study was designed to examine the effects of long-term treatment with the SGLT2 inhibitor, canagliflozin, on bone biomarkers, bone microarchitecture and material strength of bone in a mouse model of diabetic bone disease. Consistent with our previous reports $(7,10)$, deficits in both cortical and trabecular bone microarchitecture and bone strength in the femur were demonstrated in untreated diabetic animals. Additionally, bone quality and strength of diabetic vertebrae were compromised. Yet, while treatment of diabetic mice for 10 weeks with canagliflozin did improve blood glucose levels by $~ 35 \%$ throughout therapy, this degree of glucose improvement did not repair diabetes-related bone deficits, or improve fracture resistance of either the femur or L6 vertebra. Instead, drug treatment of diabetic mice was associated with increased bone resorption. Moreover, drug treatment of control mice was associated with increased calciuria, evidence of metaphyseal (trabecular) bone deterioration, and an increase in FGF23, possibly suggesting either a subclinical compensatory increase in PTH action in response to urine calcium losses, or a physiological consequence of canagliflozin-induced phosphate reabsorption.

In fact, the observed changes in renal gene expression (Figure 3) of CYP27B1 ( 11-fold increase) and SLC34A1 ( 30\% decrease) in diabetic mice are as expected in a state of hyperparathyroidism, and are in keeping with the significant increase in serum PTH observed in diabetic mice (Figure 2A). These findings likely reflect acute physiological changes intended to offset the marked increase in urinary calcium excretion resulting from hyperglycemia-mediated osmotic diuresis in the diabetic groups. In contrast, while drug treatment of control mice did increase urinary calcium loss, relative to non-treated control animals, the magnitude of effect was not sufficient to significantly change mean serum PTH levels, or to influence gene expression of CYP27B1 or SLC34A1. Nevertheless, a modest, but significant increase in mean FGF23 concentration in the CONT+CANA group was detected, perhaps inferring a PTH-dependent osteocyte response to canagliflozin-induced increases in $\mathrm{Na}^{+}$-Phosphate reabsorption in the renal proximal tubule.

It could be argued that a canagliflozin-induced 35\% drop in blood glucose, alone, was insufficient to rectify the skeletal consequences of hyperglycemia, and that nearernormalization of glycemic control, perhaps with higher drug doses, would be required. However, sub-maximal improvement in blood glucose levels is in accordance with the expected therapeutic efficacy seen in human clinical trials. For example, a meta-analysis of randomized trials demonstrated that dapagliflozin reduced fasting plasma glucose only 18.28 $\mathrm{mg} / \mathrm{dL}$, when given as adjunct therapy to persons with T2D (15). Also, urinary glucose excretion during selective SGLT2-inhibitor treatment is only 30-40\% of maximum in human studies $(16,17)$, possibly due to compensatory glucose reuptake by alternate glucose transporters. Correspondingly, studies comparing mice with an SGLT2 (slc5a2) single gene deletion to those with SGLT1 (slc5a1)/SGLT2 double gene knockout (DKO) suggest that renal SGLT1 in db/db mice has the capacity to reabsorb $\sim 70 \%$ of filtered glucose when SGLT2 is non-functional $(16,18)$, again consistent with incomplete glucose improvement, as we report. Intrinsically, our study design was limited to the use of canagliflozin as monotherapy; further investigation would be needed to determine whether a multi-drug therapy, including SGLT2 inhibitors at comparable or higher doses, could provide a level of 
glycemic improvement sufficient to prevent diabetic bone disease without undesirable consequences.

Alternatively, it could be suggested that canagliflozin, as an SGLT2 inhibitor, could impose a direct, detrimental pharmacological effect on bone cells themselves, either in the diabetic or non-diabetic mice, independent of the metabolic milieu. This hypothesis would, however, be dependent upon the expression of SGLT2 in bone cells. To our knowledge, while studies have demonstrated that high affinity glucose transporters (GLUTs) are expressed by osteoblasts (GLUT1 and GLUT3) and osteoclasts (Glut1) (22, 23), no information is currently available demonstrating expression of sodium-dependent glucose cotransporters (i.e. SGLT1 and SGLT2) in these cell types. Moreover, several studies show that expression of SGLT2 is limited to the brush border membrane of the proximal tubule cells of the kidney in rodents (19) and humans $(20,21)$. While the current study is limited in its ability to directly address this possibility, future studies could examine the expression of SGLT subtypes in bone cells, to determine if glucose transport in bone is affected by treatment with drugs such as canagliflozin.

The potential effects of SGLT2 inhibitors on healthy bone are of particular interest, given that the use of SGLT2 inhibitor therapy for therapeutic weight loss in humans has been proposed. In preclinical models of obesity, including Diet-induced obese (DIO) mice (22) and Zucker fatty (ZF) rats (22), SGLT2 inhibition for 3-4 weeks reduced body weight gain in treated rodents (22). Dual SGLT1/SGLT2 inhibition for 4 weeks has also been shown to significantly reduce body weight gain in lean animal models, including Sprague-Dawley rats and beagle dogs (23). Clinically, a recently published pilot study of the SGLT2 inhibitor, remogliflozin etabonate in obese non-diabetic individuals demonstrated a significant reduction in leptin/adiponectin ratio (a measure of insulin resistance) after 8 weeks of therapy (24), implying an improvement in the metabolic health of these individuals. In light of our findings which suggest possible drug-induced metaphyseal deficits in non-diabetic mice, it may become important to monitor the impact of longer-term SGLT2 inhibition on bone in all clinical conditions in which it is used.

\subsection{Conclusions}

In male diabetic DBA/2J mice, a sustained, $\sim 35 \%$ therapeutic improvement in blood glucose control was achievable with long-term treatment with canagliflozin, given at doses of 17-20 $\mathrm{mg} / \mathrm{kg} /$ day. However, diabetic bone disease, as was evident in this model, was not favorably affected by this drug treatment, perhaps due to insufficient glycemic improvement. Instead, in control mice, long-term exposure to SGLT2 inhibition was associated with adverse effects on the trabecular compartment of bone, along with hypercalciuria. Our findings would suggest that as the clinical indications for SGLT2 inhibitor therapy expand, care should be taken to monitor skeletal health in persons receiving this class of drugs.

\section{Acknowledgments}

This work was supported by grants from the Children's University Medical Group fund of the Arkansas Children's Hospital Research Institute (to K.M.T.), the Arkansas Biosciences Institute (to J.L.F.), and in part by National 
Institutes of Health Grants R01DK055653 (to J.L.F.), and C06RR16517 (to ACHRI), as well as the Department of Veterans Affairs, Veterans Health Administration, Office of Research and Development 1I01BX001018 (to J.S.N.).

\section{References}

1. Vestergaard P. Discrepancies in bone mineral density and fracture risk in patients with type 1 and type 2 diabetes--a meta-analysis. Osteoporosis international : a journal established as result of cooperation between the European Foundation for Osteoporosis and the National Osteoporosis Foundation of the USA. 2007; 18(4):427-44. doi: 10.1007/s00198-006-0253-4. PubMed PMID: 17068657.

2. Neumann T, Samann A, Lodes S, Kastner B, Franke S, Kiehntopf M, Hemmelmann C, Lehmann T, Muller UA, Hein G, Wolf G. Glycaemic control is positively associated with prevalent fractures but not with bone mineral density in patients with Type 1 diabetes. Diabetic medicine : a journal of the British Diabetic Association. 2011; 28(7):872-5. doi: 10.1111/j.1464-5491.2011.03286.x. PubMed PMID: 21395677. [PubMed: 21395677]

3. Nigro SC, Riche DM, Pheng M, Baker WL. Canagliflozin, a novel SGLT2 inhibitor for treatment of type 2 diabetes. The Annals of pharmacotherapy. 2013; 47(10):1301-11. doi: 10.1177/1060028013503626. PubMed PMID: 24259694. [PubMed: 24259694]

4. Perkins BA, Cherney DZ, Partridge H, Soleymanlou N, Tschirhart H, Zinman B, Fagan NM, Kaspers S, Woerle HJ, Broedl UC, Johansen OE. Sodium-glucose cotransporter 2 inhibition and glycemic control in type 1 diabetes: results of an 8-week open-label proof-of-concept trial. Diabetes care. 2014; 37(5):1480-3. doi: 10.2337/dc13-2338. PubMed PMID: 24595630. [PubMed: 24595630]

5. Sands AT, Zambrowicz BP, Rosenstock J, Lapuerta P, Bode BW, Garg SK, Buse JB, Banks P, Heptulla R, Rendell M, Cefalu WT, Strumph P. Sotagliflozin, a Dual SGLT1 and SGLT2 Inhibitor, as Adjunct Therapy to Insulin in Type 1 Diabetes. Diabetes care. 2015 doi: 10.2337/dc14-2806. PubMed PMID: 26049551.

6. Taylor SI, Blau JE, Rother KI. Possible adverse effects of SGLT2 inhibitors on bone. The lancet Diabetes \& endocrinology. 2015; 3(1):8-10. doi: 10.1016/S2213-8587(14)70227-X. PubMed PMID: 25523498. [PubMed: 25523498]

7. Fowlkes JL, Nyman JS, Bunn RC, Cockrell GE, Wahl EC, Rettiganti MR, Lumpkin CK Jr, Thrailkill KM. Effects of long-term doxycycline on bone quality and strength in diabetic male DBA/2J mice. Bone reports. 2015; 1:16-9. doi: 10.1016/j.bonr.2014.10.001. PubMed PMID: 25685827; PubMed Central PMCID: PMC4324548. [PubMed: 25685827]

8. Quarles LD. Role of FGF23 in vitamin D and phosphate metabolism: implications in chronic kidney disease. Experimental cell research. 2012; 318(9):1040-8. doi: 10.1016/j.yexcr.2012.02.027. PubMed PMID: 22421513; PubMed Central PMCID: PMC3336874. [PubMed: 22421513]

9. Fowlkes JL, Bunn RC, Liu L, Wahl EC, Coleman HN, Cockrell GE, Perrien DS, Lumpkin CK Jr, Thrailkill KM. Runt-related transcription factor 2 (RUNX2) and RUNX2-related osteogenic genes are down-regulated throughout osteogenesis in type 1 diabetes mellitus. Endocrinology. 2008; 149(4):1697-704. doi: 10.1210/en.2007-1408. PubMed PMID: 18162513; PubMed Central PMCID: PMC2276714. [PubMed: 18162513]

10. Nyman JS, Even JL, Jo CH, Herbert EG, Murry MR, Cockrell GE, Wahl EC, Bunn RC, Lumpkin CK Jr, Fowlkes JL, Thrailkill KM. Increasing duration of type 1 diabetes perturbs the strengthstructure relationship and increases brittleness of bone. Bone. 2011; 48(4):733-40. doi: 10.1016/ j.bone.2010.12.016. PubMed PMID: 21185416; PubMed Central PMCID: PMC3062641. [PubMed: 21185416]

11. Brown IR, McBain AM, Chalmers J, Campbell IW, Brown ER, Lewis MJ. Sex difference in the relationship of calcium and magnesium excretion to glycaemic control in type 1 diabetes mellitus. Clinica chimica acta; international journal of clinical chemistry. 1999; 283(1-2):119-28. PubMed PMID: 10404736.

12. Isidro ML, Ruano B. Bone disease in diabetes. Current diabetes reviews. 2010; 6(3):144-55. PubMed PMID: 20380629. [PubMed: 20380629] 
13. Malone JI, Lowitt S, Duncan JA, Shah SC, Vargas A, Root AW. Hypercalciuria, hyperphosphaturia, and growth retardation in children with diabetes mellitus. Pediatrics. 1986; 78(2):298-304. PubMed PMID: 3488537. [PubMed: 3488537]

14. Santer R, Calado J. Familial renal glucosuria and SGLT2: from a mendelian trait to a therapeutic target. Clinical journal of the American Society of Nephrology : CJASN. 2010; 5(1):133-41. doi: 10.2215/CJN.04010609. PubMed PMID: 19965550. [PubMed: 19965550]

15. Musso G, Gambino R, Cassader M, Pagano G. A novel approach to control hyperglycemia in type 2 diabetes: sodium glucose co-transport (SGLT) inhibitors: systematic review and meta-analysis of randomized trials. Annals of medicine. 2012; 44(4):375-93. doi: 10.3109/07853890.2011.560181. PubMed PMID: 21495788. [PubMed: 21495788]

16. Powell DR, DaCosta CM, Gay J, Ding ZM, Smith M, Greer J, Doree D, Jeter-Jones S, Mseeh F, Rodriguez LA, Harris A, Buhring L, Platt KA, Vogel P, Brommage R, Shadoan MK, Sands AT, Zambrowicz B. Improved glycemic control in mice lacking Sglt1 and Sglt2. American journal of physiology Endocrinology and metabolism. 2013; 304(2):E117-30. doi: 10.1152/ajpendo. 00439.2012. PubMed PMID: 23149623. [PubMed: 23149623]

17. Liu JJ, Lee T, DeFronzo RA. Why Do SGLT2 inhibitors inhibit only 30-50\% of renal glucose reabsorption in humans? Diabetes. 2012; 61(9):2199-204. doi: 10.2337/db12-0052. PubMed PMID: 22923645; PubMed Central PMCID: PMC3425428. [PubMed: 22923645]

18. Jurczak MJ, Lee HY, Birkenfeld AL, Jornayvaz FR, Frederick DW, Pongratz RL, Zhao X, Moeckel GW, Samuel VT, Whaley JM, Shulman GI, Kibbey RG. SGLT2 deletion improves glucose homeostasis and preserves pancreatic beta-cell function. Diabetes. 2011; 60(3):890-8. doi: 10.2337/db10-1328. PubMed PMID: 21357472; PubMed Central PMCID: PMC3046850. [PubMed: 21357472]

19. Sabolic I, Vrhovac I, Eror DB, Gerasimova M, Rose M, Breljak D, Ljubojevic M, Brzica H, Sebastiani A, Thal SC, Sauvant C, Kipp H, Vallon V, Koepsell H. Expression of Na+-D-glucose cotransporter SGLT2 in rodents is kidney-specific and exhibits sex and species differences. American journal of physiology Cell physiology. 2012; 302(8):C1174-88. doi: 10.1152/ajpcell. 00450.2011. PubMed PMID: 22262063; PubMed Central PMCID: PMC3774553. [PubMed: 22262063]

20. Vrhovac I, Balen Eror D, Klessen D, Burger C, Breljak D, Kraus O, Radovic N, Jadrijevic S, Aleksic I, Walles T, Sauvant C, Sabolic I, Koepsell H. Localizations of Na-D-glucose cotransporters SGLT1 and SGLT2 in human kidney and of SGLT1 in human small intestine, liver, lung, and heart. Pflugers Archiv : European journal of physiology. 2014 doi: 10.1007/ s00424-014-1619-7. PubMed PMID: 25304002.

21. Chen J, Williams S, Ho S, Loraine H, Hagan D, Whaley JM, Feder JN. Quantitative PCR tissue expression profiling of the human SGLT2 gene and related family members. Diabetes therapy : research, treatment and education of diabetes and related disorders. 2010; 1(2):57-92. doi: 10.1007/s13300-010-0006-4. PubMed PMID: 22127746; PubMed Central PMCID: PMC3138482.

22. Liang Y, Arakawa K, Ueta K, Matsushita Y, Kuriyama C, Martin T, Du F, Liu Y, Xu J, Conway B, Conway J, Polidori D, Ways K, Demarest K. Effect of canagliflozin on renal threshold for glucose, glycemia, and body weight in normal and diabetic animal models. PloS one. 2012; 7(2):e30555. doi: 10.1371/journal.pone.0030555. PubMed PMID: 22355316; PubMed Central PMCID: PMC3280264. [PubMed: 22355316]

23. Powell DR, DaCosta CM, Smith M, Doree D, Harris A, Buhring L, Heydorn W, Nouraldeen A, Xiong W, Yalamanchili P, Mseeh F, Wilson A, Shadoan M, Zambrowicz B, Ding ZM. Effect of LX4211 on glucose homeostasis and body composition in preclinical models. The Journal of pharmacology and experimental therapeutics. 2014; 350(2):232-42. doi: 10.1124/jpet.114.214304. PubMed PMID: 24849925. [PubMed: 24849925]

24. Napolitano A, Miller S, Murgatroyd P, Hussey E, Dobbins R, Bullmore E, Nunez D. Exploring glycosuria as a mechanism for weight and fat mass reduction. A pilot study with remogliflozin etabonate and sergliflozin etabonate in healthy obese subjects. J Clin Trans Endocrinol. 2014; 1:e3-e8. 


\section{Highlights}

- Deficits in cortical and trabecular bone microarchitecture and in bone strength develop in untreated diabetic male DBA/2J mice.

- In these mice, treatment with the SGLT2 inhibitor, canagliflozin, for 10 weeks improved blood glucose levels by $\sim 35 \%$.

- Despite blood glucose improvement, canagliflozin treatment of diabetic mice did not improve diabetes-related bone deficits.

- Canagliflozin exposure may adversely modify skeletal tissues in both diabetic and non-diabetic male DBA/2J mice, possibly via increased bone resorption. 

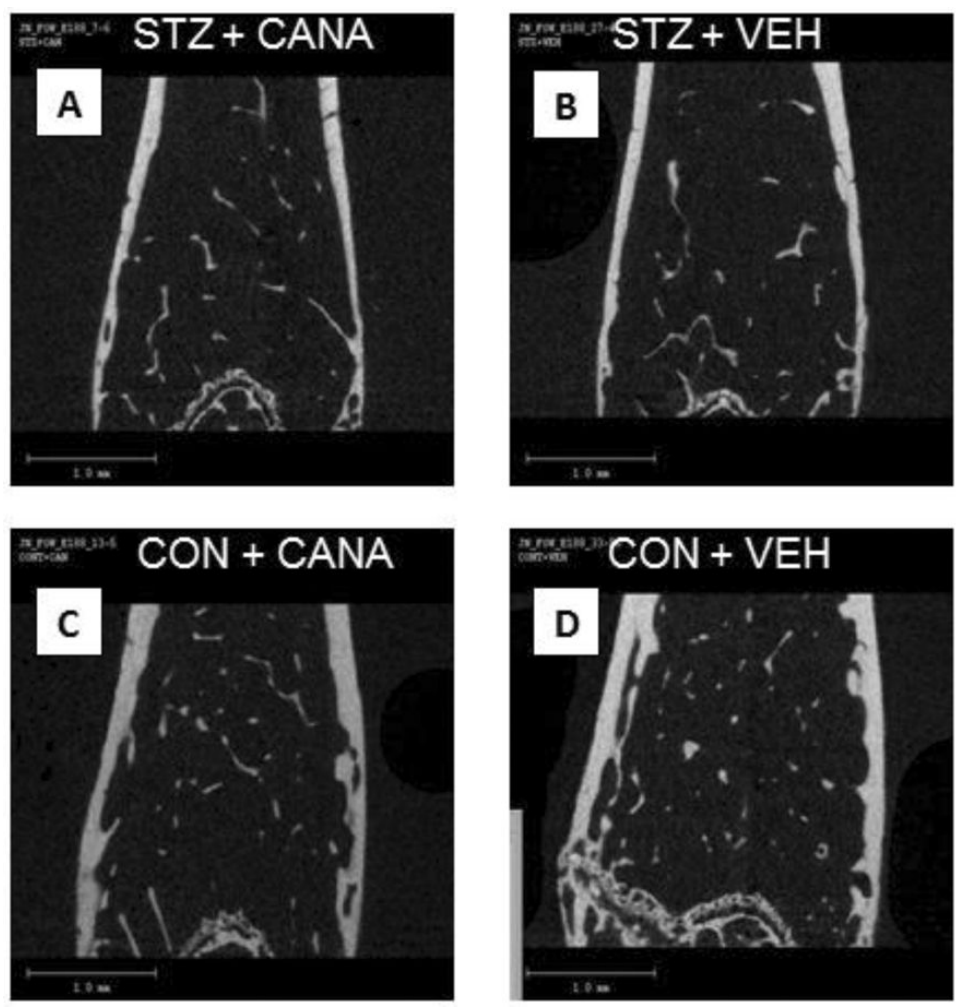

Figure 1. Representative $\boldsymbol{\mu C T}$ images

A representative $\mu \mathrm{CT}$ image from each of the four groups is shown; STZ+CANA (A), STZ +VEHICLE (B), CONTROL+CANA (C), and CONTROL+VEHICLE (D). Significant deficits in both cortical and trabecular bone were evident in mice with STZ-induced diabetes (A, B), compared with control mice (D). Specifically, as shown in these images, hyperglycemic mice exhibited decreased trabecular number (Tb.N) and increased trabecular spacing (Tb.Sp); decreased cortical thickness (Ct.Th) and increased cortical porosity (Ct.Po) can also be seen. Canagliflozin treatment did not improve diabetic bone disease, despite blood glucose reduction (A). In contrast, drug treatment of control mice (C) reduced trabecular bone volume fraction (BV/TV), Tb.N and trabecular tissue mineral density (Tb.TMD) while increasing Tb.Sp, compared to untreated control mice (D). 

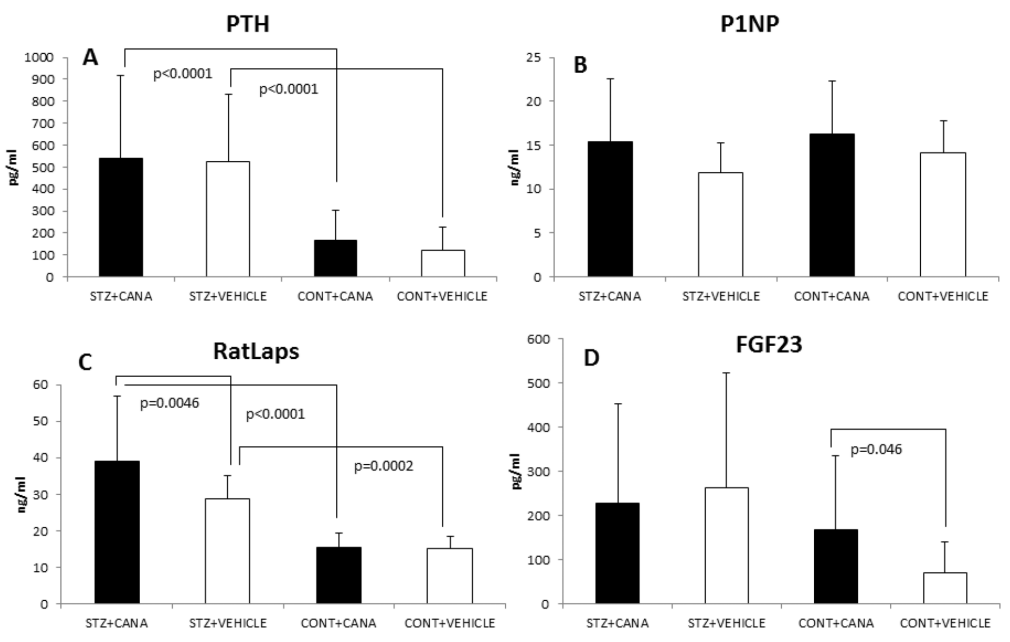

Figure 2. Bone biomarker analyses

A comparison of serum bone biomarker concentrations (mean $\pm \mathrm{SD}$ ) across the four groups is shown; STZ+CANA, STZ+VEHICLE, CONTROL+CANA, CONTROL+VEHICLE.

Pertinent, statistically significant between-group differences (and p-values) are delineated. Abbreviations: (A) PTH, parathyroid hormone; (B) P1NP, procollagen type $1 \mathrm{~N}$-terminal propeptide; (C) RatLAPS, C-terminal telopeptides of type I collagen; (D) FGF23, fibroblast growth factor-23. 

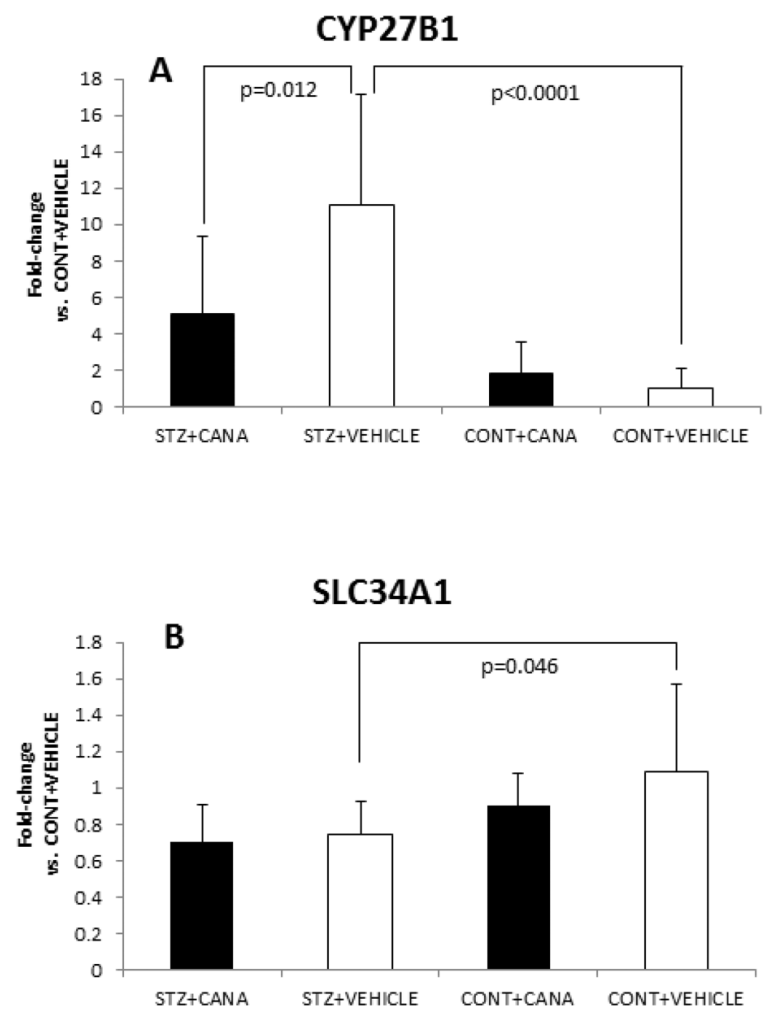

Figure 3. CYP27B1 and SLC34A1 gene expression

Differences (mean $\pm \mathrm{SD}$ ) in renal gene expression (fold-change vs. CONT+VEHICLE) for (A) CYP27B1 and (B) SLC34A1 genes are shown. The product of the CYP27B1 gene, 25hydroxyvitamin D-1a-hydroxylase, functions to synthesize $1,25-(\mathrm{OH})_{2}$ vitamin $\mathrm{D} 3$ from 25- $(\mathrm{OH})$ vitamin D3; CYP27B1 gene expression is upregulated in response to PTH and to hypocalcemia. The SLC34A1 gene encodes for the renal sodium-phosphate co-transporter, responsible for phosphate reabsorption in the proximal tubule. Co-transporter function is reduced by PTH, contributing to phosphaturia. Statistically significant between-group differences (and p-values) are delineated, showing a significant increase in CYP27B1 and decrease in SLC34A1 in diabetic mice (STZ+VEHICLE), which is in keeping with the increase in mean serum PTH observed in diabetic mice (Figure 2A). 


\section{Table 1}

Phenotypic parameters (top); and selected properties of bone (femur metaphysis, femur diaphysis, L6 vertebra), as determined by $\mathrm{uCT}$, three-point bending and compression testing.

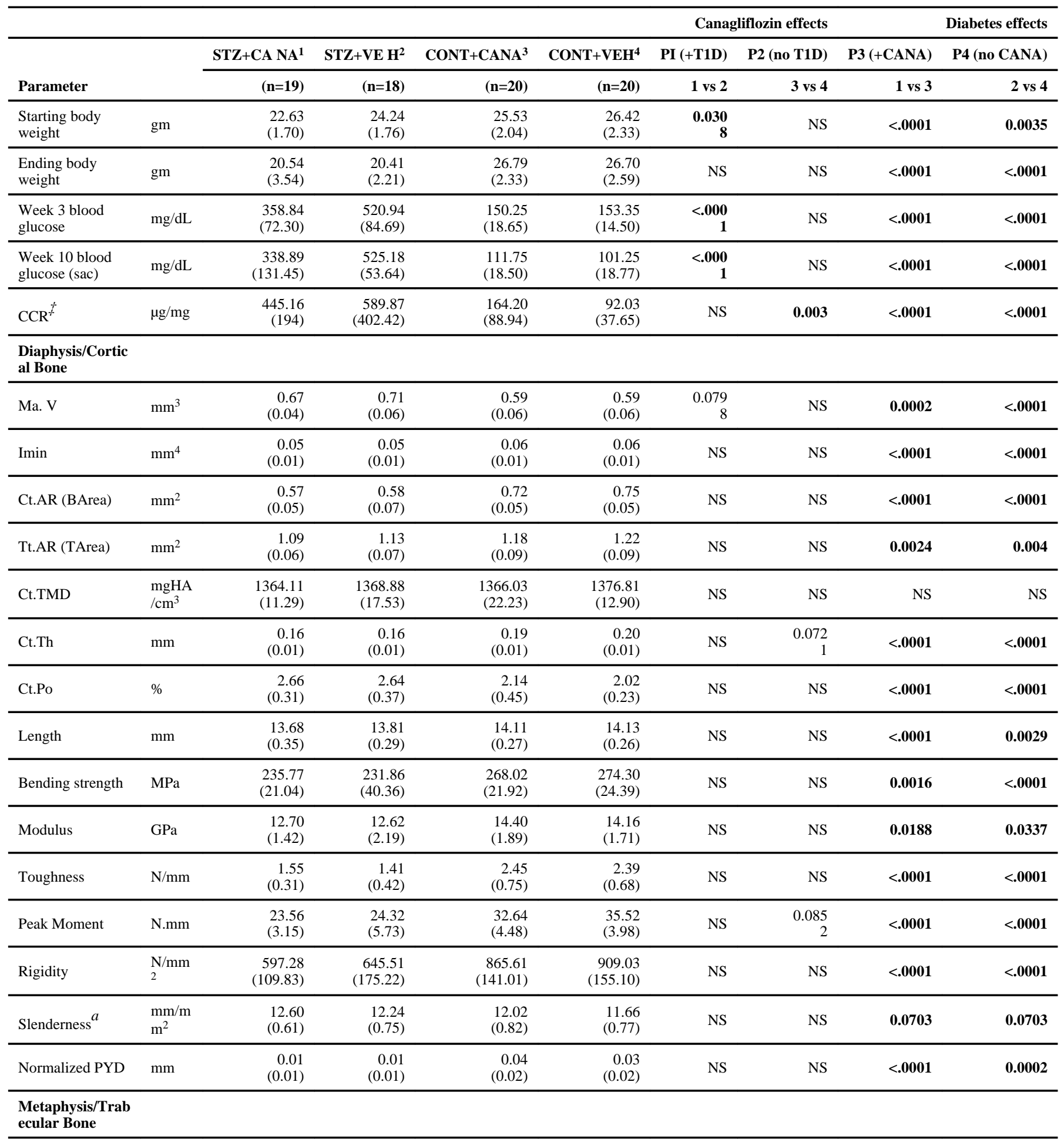




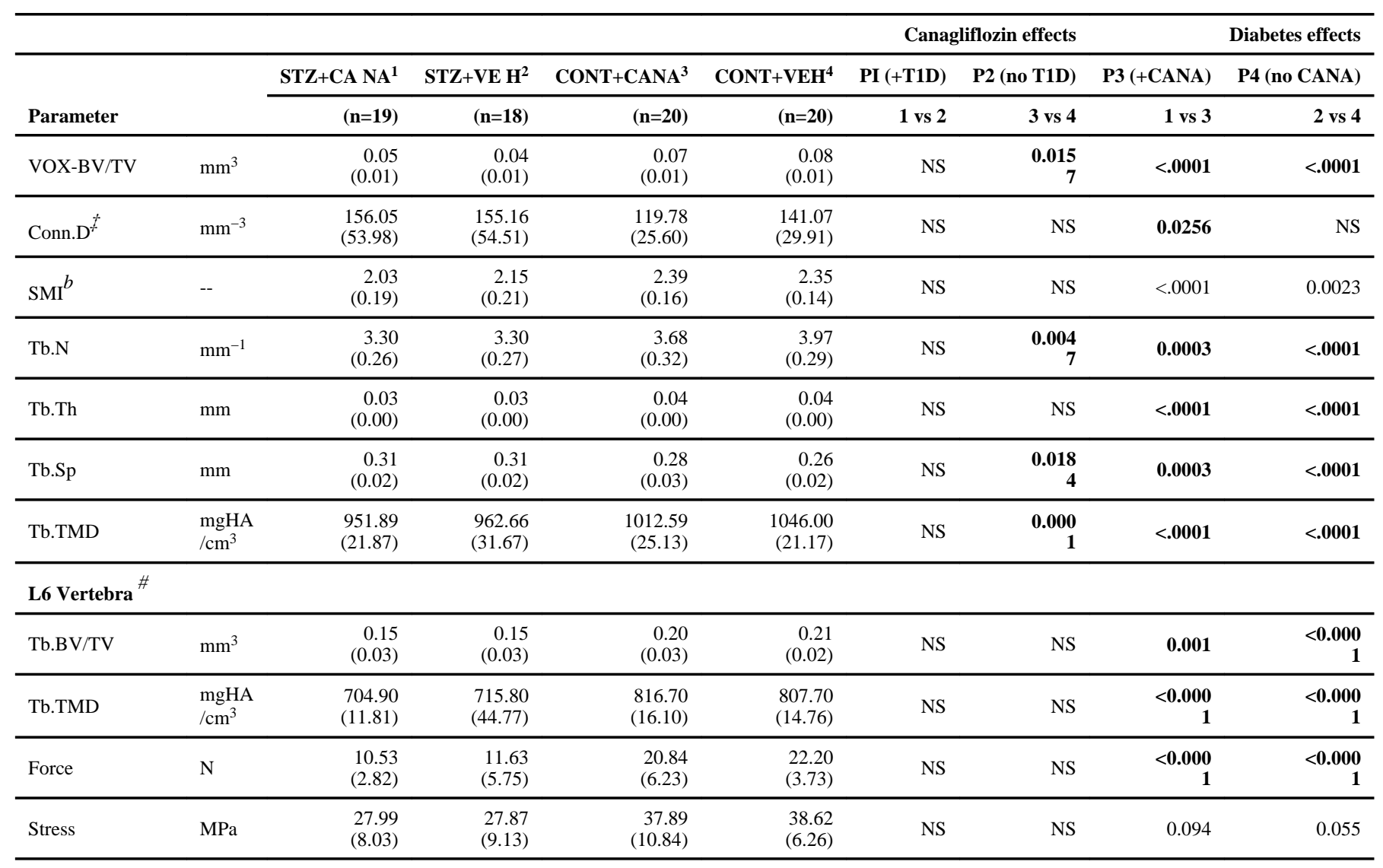

A comparison of all parameters (mean $\pm \mathrm{SD}$ ) between the four groups is shown. Pairwise p-values are indicated, as follows: PI, STZ+CANA vs. STZ+VEH; P2, CONT+CANA vs. CONT+VEH; P3, STZ+CANA vs CONT+CANA; P4, STZ+VEH vs. CONT+VEH.

Abbreviations: CCR, urine Calcium to Creatinine ratio; Alphabetically listed: BV/TV, bone volume/tissue volume; Conn.D, connectivity density; Ct.Ar, cortical area; Ct.Po, cortical porosity; Ct.Th, cortical thickness; Ct.TMD, cortical tissue mineral density; Force, peak force in compression; Imin, minimal moment of inertia; Ma.V, marrow volume; PYD, post yield deflection; SMI, structural model index; Stress, peak force per area; Tb.N, trabecular number; Tb.Sp, trabecular separation; Tb.Th, trabecular thickness; Tb.TMD, trabecular tissue mineral density; Tt.Ar, total crosssectional area. Significant differences $(p<0.05)$ are highlighted in bold font; $p<0.10$ are also shown. NS $=$ no significant difference.

${ }^{a}$ Slenderness is the ratio of the length to the total cross-sectional area of the femur mid-shaft.

${ }^{b}$ Structural model index characterizes the shape of trabecular bone (1: plate-like; 3: rod-like).

*Variables were log-transformed due to violation of the normality assumption.

\# Vertebral analyses conducted on $\mathrm{n}=10$ mice per group. 